\title{
Premature Atherosclerosis Long After Kawasaki Disease
}

\author{
Nobutaka Noto and Tomoo Okada \\ Department of Pediatrics and Child Health, Nihon University School of Medicine, Tokyo, \\ Japan
}

\section{Introduction}

Kawasaki disease (KD) is a systemic vasculitis of unknown etiology in infants and children. First described in Japan in 1967, KD has been described worldwide among children of all races and ethnicities (Kawasaki, 1967). More than 4000 hospitalizations associated with KD were reported in 2000 and KD is the leading cause of acquired heart disease in childhood in the United State (Taubert et al., 1991). In Japan, the national survey of KD has performed every 2 years since 1970. From the most recent 20th survey dealt with the years 2007 and 2008 , the number of new cases of KD in those years was 11,581 and 11,756, respectively. As of 2008, the total number of patients since 1970 was 249,019 (Nakamura et al., 2010). Therefore, patients diagnosed with KD in the sixties and seventies have already reached adulthood. The increased incidence of young adults who experience KD during childhood has been accompanied by a new problem of an association between post-KD lesions and atherosclerosis.

In this chapter, we would like to review and verify with regarding to the propensity of "Premature atherosclerosis long after Kawasaki disease" from several cutting edges such as epidemiologic features, dyslipidemia, non-invasive diagnostic methods for evaluating atherosclerosis such as flow-mediated dilatation (FMD) of brachial artery, carotid intimamedia thickness (IMT), pulse wave velocity (PWV), invasive diagnostic method such as intravascular ultrasound imaging (IVUS) and pathological features along with the findings of our recent studies.

\section{Epidemiology of Kawasaki disease}

The incidence rate and number of patients with KD in Japan continue to increase. The 20th nationwide survey of KD in Japan was conducted in 2009, and included patients treated for the disease in 2007 and 2008 (Nakamura et al., 2010). Hospitals specializing in pediatrics, and hospitals with pediatric departments and 100 or more beds, were asked to report all patients with KD during the 2 survey years. From a total of 1540 departments and hospitals, 23,337 patients (11 581 in 2007 and 11756 in 2008) were reported: 13,523 boys and 9814 girls. The annual incidence rates were 215.3 and 218.6 per 100,000 children aged 0-4 years in 2007 and 2008, respectively. This incidence was approximately 10 to 25 times higher than that of the United States and the United Kingdom. These were the highest annual KD incidence 
rates ever recorded in Japan. The age-specific incidence rate showed a monomodal distribution with a peak at age 9-11 months. The prevalence of both cardiac lesions during the acute phase of the disease and cardiac sequelae were higher among infants and older age groups. During the acute phase, 2577 (11.0\%) patients had a cardiac lesion(s): 58 (0.25\%) had giant coronary aneurysms, $282(1.21 \%)$ had coronary aneurysms less than $8 \mathrm{~mm}$ in diameter, $1992(8.54 \%)$ had coronary dilatations, 8 (0.03\%) had coronary stenoses, $3(0.01 \%)$ had myocardial infarction, and $383(1.64 \%)$ had valvular lesions. A total of 746 patients (3.2\%) had cardiac sequelae 1 month after the onset of KD: 59 (0.25\%) had giant coronary aneurysms, $188(0.81 \%)$ had coronary aneurysms less than $8 \mathrm{~mm}$ in diameter, $435(1.86 \%)$ had coronary dilatations, $5(0.02 \%)$ had coronary stenoses, $2(0.01 \%)$ had myocardial infarction, and $114(0.49 \%)$ had valvular lesions. The proportion of patients with cardiac sequelae has decreased year by year. The proportion was $7.0 \%$ in the 15 th nationwide survey in 1997-1998, 5.9\% in the 16th (1999-2000), 5.0\% in the 17th (2001-2002), $4.4 \%$ in the 18th (2003-2004), and $3.8 \%$ in the 19th survey. $87 \%$ of patients received intravenous immunoglobulin therapy (IVIG). Of those, $16.5 \%$ of patients received additional IVIG therapy, $5.0 \%$ of patients were treated with steroids, $0.35 \%$ of patients received infliximab, and $0.23 \%$ of patients were treated with immunosuppressive agents. Although the incidence of coronary sequelae has gradually been decreasing, the rate of severe sequelae, such as giant aneurysms, has not been decreasing as expected (Figure 1). Patients with persistent coronary aneurysms, stenoses, and regressed aneurysms have suffered the most severe arterial insult. In such patients, the possibility of early progression to atherosclerosis occurs. It is therefore important to determine the factors concerning with how to reduce the patients with severe sequelae.

\section{Fate of coronary sequelae in Kawasaki disease}

Kato et al. demonstrated the angiographic outcome of coronary aneurysms in KD (Kato et al., 1996). This fundamental work has led to the current understanding that coronary artery lesions (CALs) change markedly with time into a variety of forms. These lesions may regress, stay unchanged, progress to stenosis or obstructive lesions with or without recanalization, and on very rare occasions, rupture, develop new lesions, or expand. These structural changes may modified the early progression of atherosclerosis long after KD.

\subsection{Regression}

Many aneurysms formed in the acute phase demonstrate a shrinking tendency in the convalescent phase and thereafter. The phenomenon in which the aneurysm resolves normally and disappears on coronary angiography is described as regression, and commonly occurs 1 to 2 years after disease onset. Regression frequently occurs for small-tomedium-sized aneurysms, and $32-50 \%$ of patients with CALs (Sasaguri\& Kato, 1982) (Suzuki et al., 1994). Regression is mainly the results of smooth muscle cell infiltration and proliferation in the intima.

\subsection{Occlusion}

Thrombotic occlusion is often seen in medium-sized or large coronary aneurysms. Suzuki et al. have reported that $16 \%$ of coronary aneurysms are occluded at follow-up, $78 \%$ of those became occluded within 2 years from onset (Suzuki et al., 1997). Whereas, acute myocardial 
infarction and sudden death have been caused by coronary artery occlusion, two-thirds of cases with occlusion have no symptomatic episodes due to the consequence of postocclusion recanalization and development of collateral vessels. Tsuda et al. reported the clinical features of 50 adult patients (mean age 28 years old) who had an acute coronary syndrome caused by coronary artery lesions due to KD from 1980 to 2008 . Of the 50 patients, 43 had thrombotic occlusion of an aneurysm and 40 had giant coronary aneurysms. Based on these evidences, it is likely that patients with known aneurysms during the acute phase of KD will have some cardiovascular morbidity and mortality as young adults (Tsuda et al., 2011).

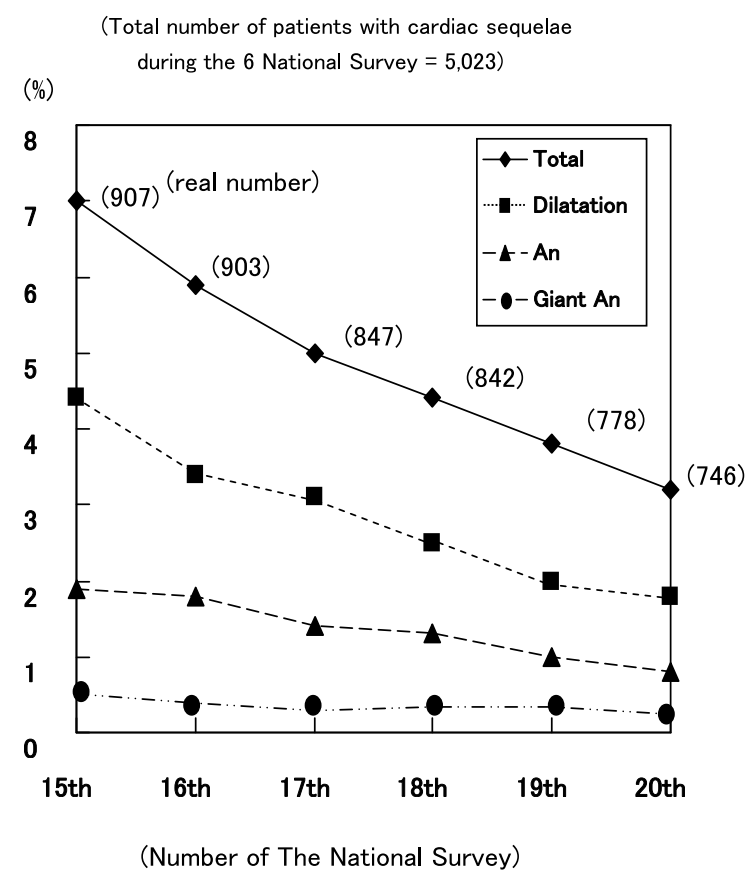

Fig. 1. Transition of the number of patients with cardiac sequelae in KD An, coronary aneurysm; Dilatation, coronary dilatation.

\subsection{Recanalization (segmental stenosis)}

New vessels after occlusion are called as segmental stenosis. Segmental stenosis has been observed in $14.8 \%$ of patients with KD sequelae, $90 \%$ of which occurred in the right coronary artery. The right coronary artery is occluded or recanalized more easily than the left coronary artery (Suzuki et al., 1997).

\subsection{Localized stenosis}

At coronary angiography, localized stenosis is reported to occur at a frequency from $12 \%$ to $4.7 \%$ (Kato et al.,1996). Localized stenosis is often seen especially in the territory of the left anterior descending coronary artery, and usually occurs in both the inflow and outflow shoulder of the aneurysm. It is caused mainly by inward luminal intimal thickening. 


\subsection{Coronary artery with no aneurysm formation}

Coronary arteries that appear normal from the onset of KD have been regarded as normal as yet. Even in coronary arteries in which there is no aneurysm formation in the acute phase, mild intimal thickening may be seen in some patients (Takahashi et al.,2001). Therefore, there is some debate about the possibility that a history of KD might be a risk factor in the progression of atherosclerosis.

\section{Dyslipidemia in Kawasaki disease}

It is well known that the process of atherosclerosis is enhanced in the presence of cardiovascular risk factors (CRFs). Undoubtedly, one of the strongest CRFs for atherosclerosis is dyslipidemia. Among patients characteristics that influence vascular health, dyslipidemia is prevalent in patients with KD with or without overt coronary artery sequelae well beyond the time that the clinical disease has resolved. Earlier studies have shown that high-density lipoprotein cholesterol (HDL-C) levels may be depressed in the acute phase of KD (Okada T et al, 1990) (Newberger JW et al., 1991). However, there is controversy whether this decrease persists over the long time. In the convalescent phase, McCrindle et al. showed that KD patients 10 to 20 years of age had lower apolipoprotein A1 levels than the healthy control subjects (McCrindle et al., 2007). Mitani et al. showed that there were no differences between patients with KD 10 years after the onset and the control subjects with regard to total cholesterol (T-C) and HDL-C levels (Mitani et al., 2005). Cheung et al. reported that KD patients around 10 years old with or without coronary artery lesion (CAL) had significantly higher levels of apolipoprotein B and lower levels of HDL-C compared with control subjects (Cheung et al., 2004). Therefore, no consensus has been reached whether dyslipidemia in KD patients over the term after clinical resolution causes a higher risk of atherosclerosis than that in the general population. In epidemiological studies of children, the best correlation with adult cholesterol levels was obtained from cholesterol levels measured during the late teen years, suggesting that subtle adverse lipoprotein profiles obtained at this age could be predictive of future dyslipidemia in young adulthood (Strong WB et al., 1992). Since young adults often become much less active once they leave school and physical activities are generally restricted in KD patients at AHA risk levels IV to $\mathrm{V}$ with giant coronary aneurysms and stenoses, it is likely that subtle risk factors cluster in individual patients. Hence, we suggest that even in subtle abnormalities of lipid profile in post-KD patients with CALs may contribute to further endothelial dysfunction and the propensity for subclinical atherosclerosis in the future.

\section{Inflammatory examinations in Kawasaki disease}

Atherosclerosis is an inflammatory disease as demonstrated in animal models and human. Clinical or subclinical inflammation of the coronary and systemic arteries may form the substrate for long-term functional and structural abnormalities and increase the risk of premature atherosclerosis. Serum high-sensitivity C-reactive protein (hs-CRP) and serum amyloid-A (SAA), have recently been regarded as reliable clinical markers for the prediction of coronary events, independent of other known CRFs (Mitani et al., 2005). In fact, serum high-sensitivity C-reactive protein (hs-CRP) level in patients with CALs is significantly higher than those seen in normal age-matched control or among patients with KD without aneurysms or regressed aneurysms. C-reactive protein inhibits NO production by 
endothelial cell and increases the endothelial expression of adhesion molecules. Inflammatory mediators, such as hs-CRP, may themselves promote atherosclerosis. Therefore, we suspect that in patients with CALs chronic low grade inflammation may continue after the acute phase of KD. Whereas, several studies suggest that oxidative stress may promote endothelial dysfunction through increased production of reactive oxygen species (ROS) (Griendling et al., 2003). Urinary 8-iso-prostaglandin F2a is increased long after the onset of KD, as well as after acute phase KD. Oxidative stress, evidenced by significant higher levels of malonyldialdehyde (MDA) and hydroperoxides, is increased in KD patients with CALs compared with those in control subjects and is associated with carotid intima-media thickness and stiffness (Cheung et al., 2008).

\section{Endothelial function in Kawasaki disease}

\subsection{Non-invasive method for evaluating endothelial function}

A number of noninvasive methods have been developed to study endothelial function and structural changes suggestive of atherosclerosis. Brachial artery flow-mediated dilatation (FMD) has been studied widely and applied to varied group of patients including children. The \%FMD reflects endothelial NO-dependent vasodilatation. Decreased \%FMD reflects endothelial cell dysfunction, and a significant decrease in \%FMD is a common feature of early marker in adult atherosclerosis. Decreased \%FMD in patients with KD has been reported by many facilities (Niboshi et al., 2008). Ikemoto et al. demonstrated endothelial dysfunction, as indicated by decreased \%FMD, only among patients with persistent CALs (Ikemoto et al., 2005). Endothelial dysfunction is worst among patients with coronary artery aneurysms (Dhillon et al.,1996) (Noto et al., 2009) (Figure 2). Abnormalities of systemic endothelial function are present many years after resolution of acute KD, even in patients without detectable early coronary involvement. In contrast, McCrindle et al. reported that patients with KD, compared with healthy control subjects, had similar systemic endothelial function, assessed by \%FMD. Furthermore, FMD was not significantly related to either patient or KD characteristics (McCrindle et al., 2007). With respect to the noninvasive method investigating endothelial dysfunction after $\mathrm{KD}$, data are not consistent.

\subsection{Invasive method for evaluating endothelial function}

The coronary artery response to acetylcholine or isosorbide has been well documented as a marker for endothelial dysfunction. Isosorbide induces dilatation of arteries in an endothelium-independent manner. On the other hand, acetylcholine dilates arteries in an endothelium-dependent manner. The reaction to acetylcholine of normal-appearing coronary arteries in patients with $\mathrm{KD}$ is equivalent to that of the control. However, the response to acetylcholine of coronary arteries with aneurysms or stenoses or both is poor dilatation or even constriction (Yamakawa et al., 1998)(Iemura et al., 2000) .

\section{Diagnostic methods for assessment of atherosclerosis in Kawasaki disease}

\subsection{Carotid intima-media thickness}

Structural arterial abnormalities are indicated by cartid intima-media thickness (IMT) measured by B-mode ultrasonography. Increased IMT has been shown to reliably indicate 
the presence of atherosclerosis. In KD patients with persistent CALs, compared with control patients, the carotid arterial wall has been reported to have a higher IMT and lower distensibility (Noto et al., 2001\&2007) (Figure 3). Carotid IMT, expressed as both unadjusted dimension and z-score, was greater among patients with KD than control subjects; within the KD group, patients with CALs had greater carotid IMT than those without CALs (Della Pozza et al., 2007). Cheung et al. found increased carotid IMT even among patients with KD with normal coronary arteries, compared with control subjects (Cheung et al., 2007). However, some investigators have found no difference in carotid IMT between patients with KD and control subjects, consistent with the hypothesis that functional abnormalities might precede those of structure (McCrindle et al., 2007).
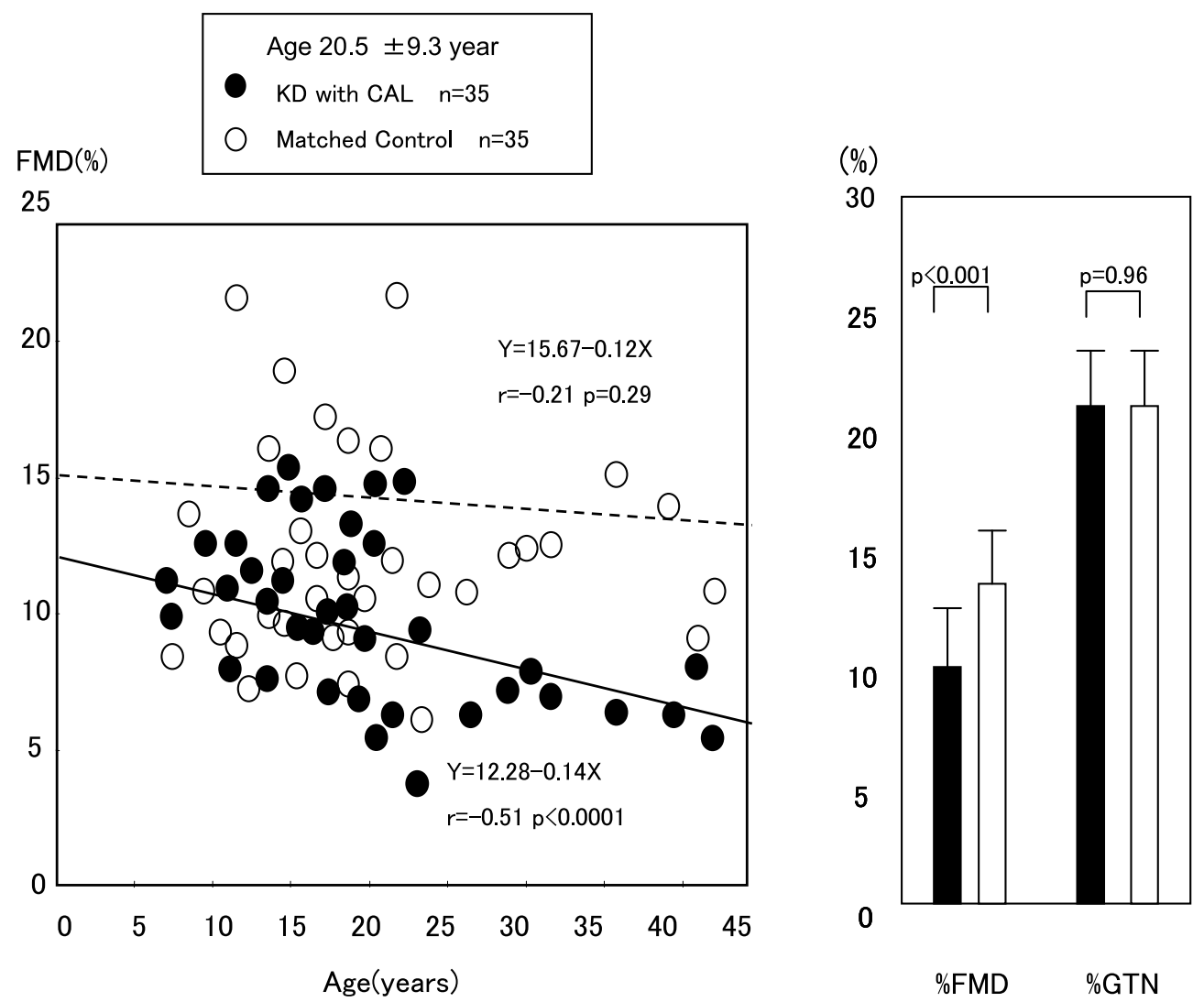

Fig. 2. Endothelial dysfunction in KD patients with CALs . (Left) Scatter plot of FMD\% versus age in the subjects with $\mathrm{KD}(\bullet)$ and the control subjects(०). Regression lines for subjects with KD and controls are represented by a solid and dashed line, respectively. (Right) \%FMD and \%GTN in KD (black bar) and control subjects (white bar). FMD, flow mediated dilatation;

GTN, sublingual glyceryl trinitrate. 

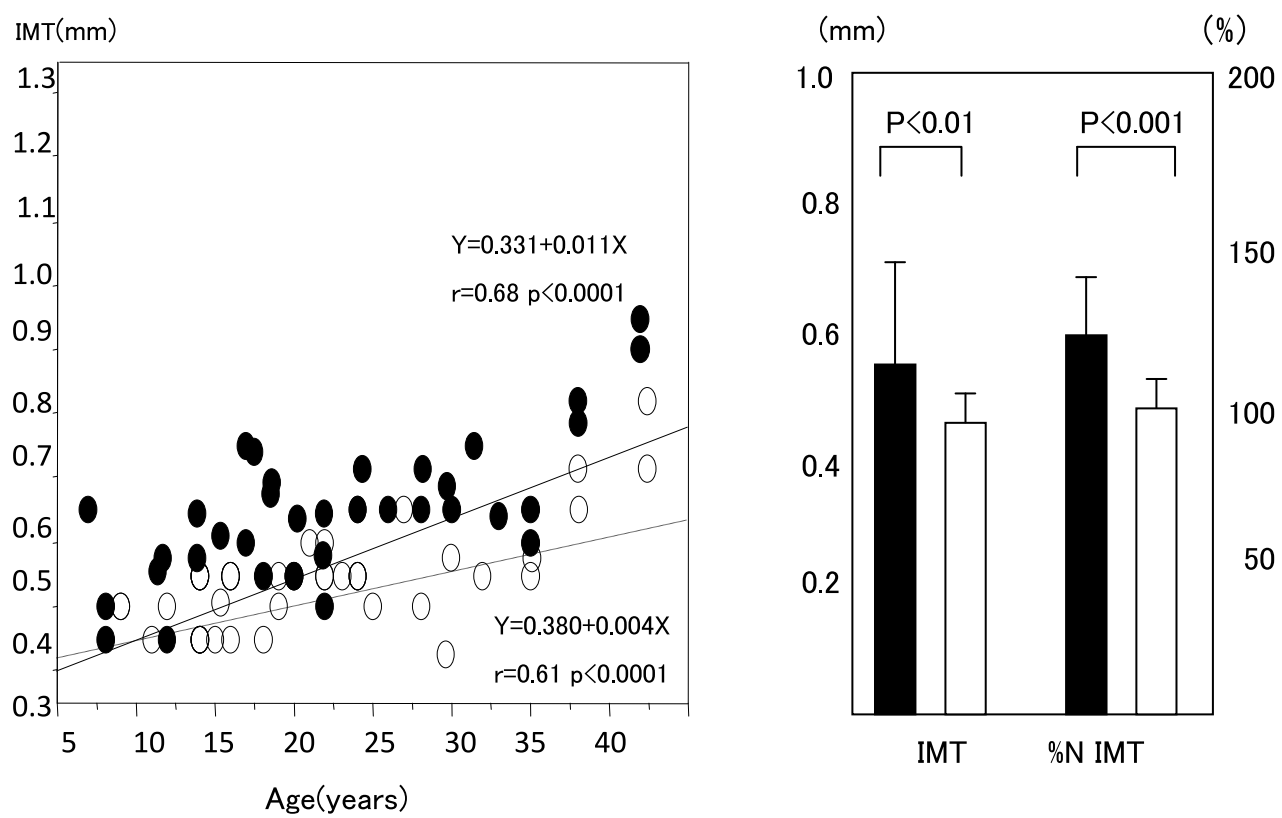

Fig. 3. Intima-media thickness (IMT) and \% normal predicted IMT (\%N IMT) in KD patients and control subjects. (Left) Scatter plot of IMT versus age for subjects with $\mathrm{KD}(\bullet)$ and control subjects (०). Regression lines for subjects with KD and controls are represented by a solid and dashed line, respectively. A significant correlation $(\mathrm{r}=0.61, \mathrm{p}<0.001)$ between age and IMT was observed. (Right) Bar graphs of IMT and \% N IMT in KD patients (black bar) and control subjects (white bar). The normal predicted IMT value (np-IMT) and percent normal predicted IMT value $(\% \mathrm{~N}$ IMT) were calculated from the following formula: $\mathrm{np}-$ $\mathrm{IMT}=0.381+0.004 \times$ age, $\% \mathrm{~N}$ IMT $=100 \times$ measured $\mathrm{IMT} / \mathrm{np}-\mathrm{IMT}$.

\subsection{Pulse wave velocity}

An alternative noninvasive method is measurement of arterial stiffness by pulse wave velocity (PWV). The PWV is simple to measure and is a powerful marker for the risk of atherosclerosis in adults. It has been reported that PWV is especially high in patients with KD who have CALs. Patients with a history of KD have a higher PWV than control subjects, moreover there is no significant difference in the PWV between the KD patients with or without CALs (Cheung et al., 2004). However, in other study PWV does not differ significantly between KD patients with normal-appearing coronary arteries and healthy controls (Ooyanagi et al., 2004). Data are conflicting.

\subsection{Intravascular ultrasound imaging}

Iemura et al have shown the evidence of persisting abnormal vascular wall morphology and vascular dysfunction at the site of regressed coronary aneurysms in patients with KD by intravascular ultrasound imaging using intracoronary infusion of acetylcholine and isosorbide dinitrate (Iemura et al., 2000). More recently, Mitani et al have demonstrated the 
plaque composition and morphology in CALs after KD using a virtual histologyintravascular ultrasound imaging (VH-IVUS). Qualitatively, the normal coronary segment had no or trivial intravascular ultrasound-visible plaque area. While, the CALs exhibited a heterogeneous plaque area with the components (fibrous, fibrofatty, necrotic core, and dense calcium) in different amounts and proportions. VH-IVUS findings in KD suggest that arteriosclerotic lesions found in CALs but not in the normal segment in patients with KD are characterized by a heterogeneous intimal area, distinct from a purely fibrotic area (Mitani et al., 2009). These findings may give new insight into the potential role of atherosclerosis in CALs long after KD.

\section{Histological examinations in Kawasaki disease}

\subsection{Pathological features}

In the acute phase of KD, histopathological investigations has shown that inflammatory cells invade the intima and destroy the internal elastic lamina and continue to infiltrated the tunica media. With inflammatory cell invasion from the adventitia, panvasculitis develops (Naoe et al., 1991).In the convalescent phase, even in clinically normal coronary artery of a child, who died of unrelated causes, pathological studies in autopsy cases showed a slightly thickened intima and the disrupted lamina interna in some parts and many a-actin-positive smooth muscle cells were observed in the intima (Suzuki et al., 2004). In fact, the lamina interna serves as a barrier for cells and macromolecules migration between the intima and the media in the vascular wall. Therefore, one can easily expect that KD patients with CALs might have a vulnerability to more easily develop atherosclerotic changes in early adulthood according to these structural alterations. Whereas, current histological studies showed that there was no significant macrophage infiltration or foamy cell formation in the intima in KD coronary arteries with localized stenosis several years after the onset of KD (Suzuki et al., 2000). Likewise, no atheromatous plaques were found in seemingly normal coronary arteries distal to aneurysmsin 10 autopsied adults KD patients (Burns et al., 1996). On the contrary, Takahashi et al. reported that 3 patients who were 15, 20, and 39 years of age had new intimal thickening, including atheroma-like bright areas and foamy macrophages in CALs (Takahashi et al., 2001). Also, Negoro et al. showed that a substantial lipid core with cholesterol crystals and macrophages was found in an atherectomy specimen from a stenotic lesion in a 32-year-old man who presented with acute coronary syndrome (Negoro et al., 2003). Due to conflicting results, we speculate that there is some heterogeneity on the propensity of atherosclerosis in individual including such as age, the latent CRFs and the given degree of structural changes after vascular remodeling in KD with CALs. At least, aneurysms are present, a history of KD in children become a risk factor for atherosclerosis later in life. Thus, a study of coronary arteries more than a decade after being afflicted with KD is greatly needed.

\subsection{Immunohistological features}

Current immunohistological studies using antibodies against vascular growth factors (GFs), such as transforming growth factor $\beta 1$, platelet-derived growth factor-A, basic fibroblast growth factor, and vascular endothelial growth factor, demonstrated that GFs were strongly expressed in the newly formed microvessels within the intima and some GFs were also 
expressed in adventitial vasa vasorum in the localized and recanalized vessels of KD children with CALs (Suzuki et al., 2000). Furthermore, vascular senescence, demonstrated as increased $\beta$-galactosidase activity, adhesion molecules and pro-inflammatory cytokines, as well as a reduction of normal physiological vascular proteins, such as eNOS, was increased in KD with CALs. These findings were more remarkable in intima as well as in vasculature of vasa vasorum (Fukazawa et al., 2007). Inevitably, adult atherosclerosis originates from the intimal side of the arteries. In contrast, arteriosclerotic changes characterized by active vascular remodeling including with luxuriant intimal proliferation and neoangiogenesis in KD with CALs may develop earlier from the both sides of the intima and adventitia of the arteries.

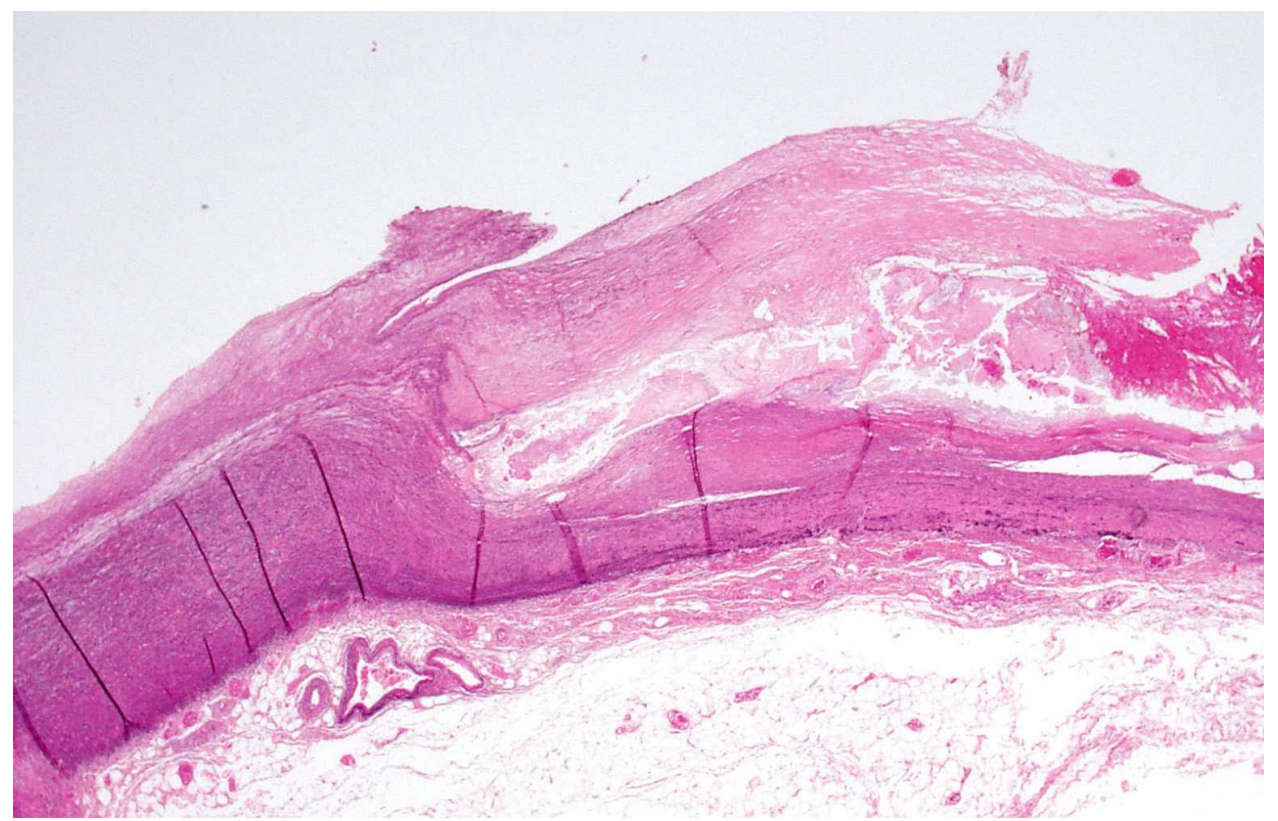

Takahashi K, et al. Pediatr Cardiol, 2001.

Fig. 4. Complicated atherosclerotic lesion in the orifice of the left coronary artery. (Elastica$\mathrm{HE}, \times 10)$. Right side is the orifice of the left coronary artery. Left side is aorta.

\section{Experimental model of coronary arteritis}

Recently, Ozawa et al. developed an animal model of KD, which is similar to the model of vasculitis in juvenile rabbits. Allergic coronary arteritis in rabbits induced by serial horse serum injections showed typical panarteritis characterized by inflammatory cell invasion of both sides from intima and adventitia, medial edema, and destruction of internal elastic lamina. When a high fat diet was being fed to this allergic arteritis rabbits models, typical atherosclerotic plaque appeared significantly (Ozawa et al., 2006). This experimental result 
suggests that post arteritis tissue may more easily develop atherosclerosis. Intriguingly, they also demonstrated the anti-inflammatory activities of $\mathrm{HMG}-\mathrm{CoA}$ reductase inhibitors against the progress of acute arteritis (Hamaoka et al., 2010).

\section{The propensity for atherosclerosis in KD}

Due to conflicting previous reports dealing with the endothelial function and non-invasive diagnostic methods in $\mathrm{KD}$, concerns have been raised as to whether $\mathrm{KD}$ patients are really at risk for premature atherosclerosis later in adulthood. Although the exact reasons for these conflicting results remain undetermined, differences in study populations, methodology, and latent CRFs including age, pubertal status, systemic inflammation may play a role. Age is of particular interest because the atherosclerotic process begins in childhood. Additionally, a strong association exists between the adverse lipoprotein levels and the initial stages of atherosclerosis in adolescents and young adults, and advanced atherosclerotic lesions are enhanced with age. We speculated that endothelial dysfunction and the propensity for subclinical atherosclerosis gradually appear during adolescence, and then rapidly increase with age, particularly in post-KD patients with CALs, who show diffuse vascular inflammation during the acute phase of KD. To test this hypothesis, we examined the relationship between age and the progression of endothelial dysfunction and subclinical atherosclerosis in case-control study using post-KD patients with CALs and healthy control subjects across a wide age range (Noto et al 2009).

\subsection{Study design}

A case-control study was performed that included 35 post-KD subjects across a wide age range (range 8-42 years) without traditional cardiovascular risk factors (CRFs) and 35 ageand sex-matched healthy control subjects (Cont). Flow-mediated dilatation (FMD) of the brachial artery induced by reactive hyperemia, intima-media thickness (IMT), and the elastic modulus (Ep) of the common carotid artery were compared between KD and Cont subjects assessed against age.

\subsection{Results}

KD subjects had slightly higher levels of BMI, lipid profile, and HbA1c than Cont subjects, but the differences were not significant. The mean IMT $(\mathrm{p}<0.001)$, age-adjusted \% normal IMT $(\% \mathrm{~N}$ IMT $)(\mathrm{p}<0.0001)$, and Ep $(\mathrm{p}<0.001)$, were significantly higher in KD than Cont subjects, and the peak FMD\% $(p<0.01)$ was significantly lower in KD than Cont subjects. There were significant correlations between FMD $\%$ and age $(r=-0.51 p<0.0001)$, IMT and age $(R=0.68 p<0.001)$, and Ep and age $(r=0.58 p<0.01)$ in $K D$ but not Cont subjects. When the difference in FMD \% between KD and matched Cont subjects $(\triangle \mathrm{FMD} \%$ ) was plotted against age, no significant relationship was found, although significant correlations between $\triangle \mathrm{IMT}$ and age $(\mathrm{r}=0.52, \mathrm{p}<0.01)$ as well as $\Delta \mathrm{Ep}$ and age $(\mathrm{r}=0.46, \mathrm{p}<0.05)$ were observed. When we defined values that were +2.0 SD over the mean control values (i.e. $\% \mathrm{~N}$ IMT $\geq 120 \%$ and or $\mathrm{Ep} \geq 50 \mathrm{KPa}$ ) as markers of subclinical atherosclerosis, 15 subjects met the criteria. Subjects over the age of 22 years were more likely to be $(O R=16.54, p=0.0001)$ subclinical atherosclerosis in this cohort (Figure 5)(Figure 6)(Figure 7). Our results suggest that endothelial dysfunction and the development of premature atherosclerosis were accelerated in adult post-KD than Cont subjects. 

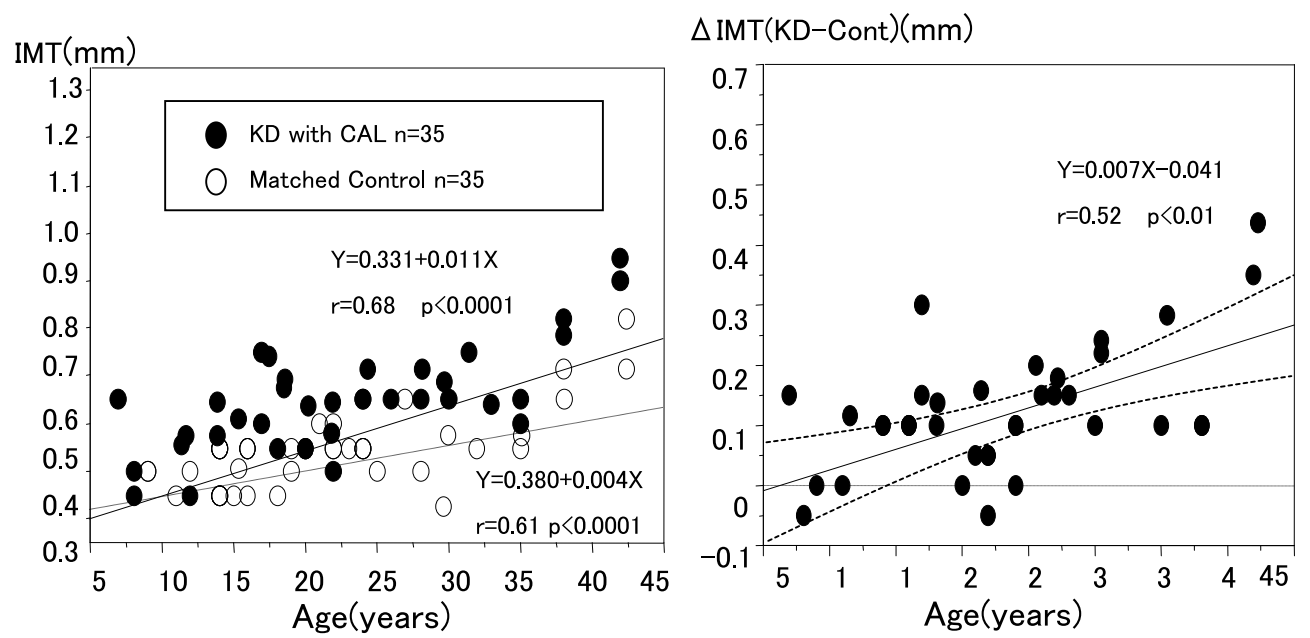

Fig. 5. Relationship between IMT and the age in KD patients and control subjects. (Left) Scatter plot of IMT versus age for subjects with $\mathrm{KD}(\bullet)$ and control subjects(०).

Regression lines for subjects with KD and controls are represented by a solid and dashed line, respectively. (Right) Difference in IMT( $\triangle \mathrm{IMT})$ and 95\% CI between subjects with KD and controls plotted against age. Mean=solid line, 95\% CI=dashed lines.
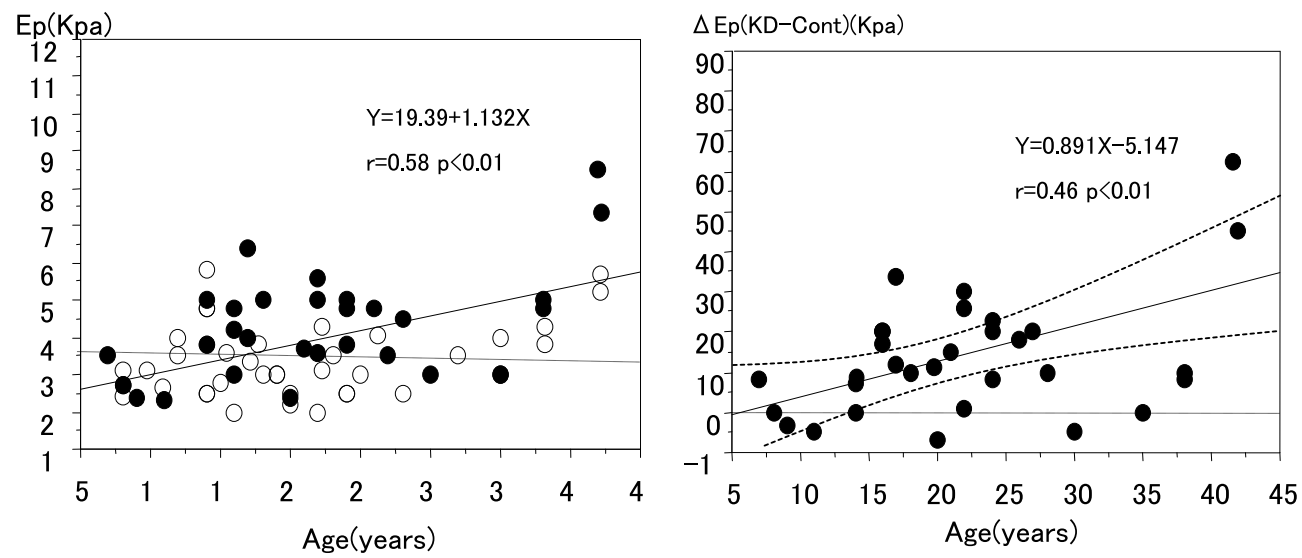

Fig. 6. Relationship between Ep and the age in KD patients and control subjects. (Left) Scatter plot of Ep versus age for subjects with $\mathrm{KD}(\bullet)$ and control subjects( $(\circ)$. Regression lines for subjects with KD and controls are represented by a solid and dashed line, respectively. (Right) Difference in $\mathrm{Ep}(\Delta \mathrm{Ep})$ and $95 \% \mathrm{CI}$ between subjects with $\mathrm{KD}$ and controls plotted against age. Mean=solid line, $95 \% \mathrm{CI}=$ dashed lines. 

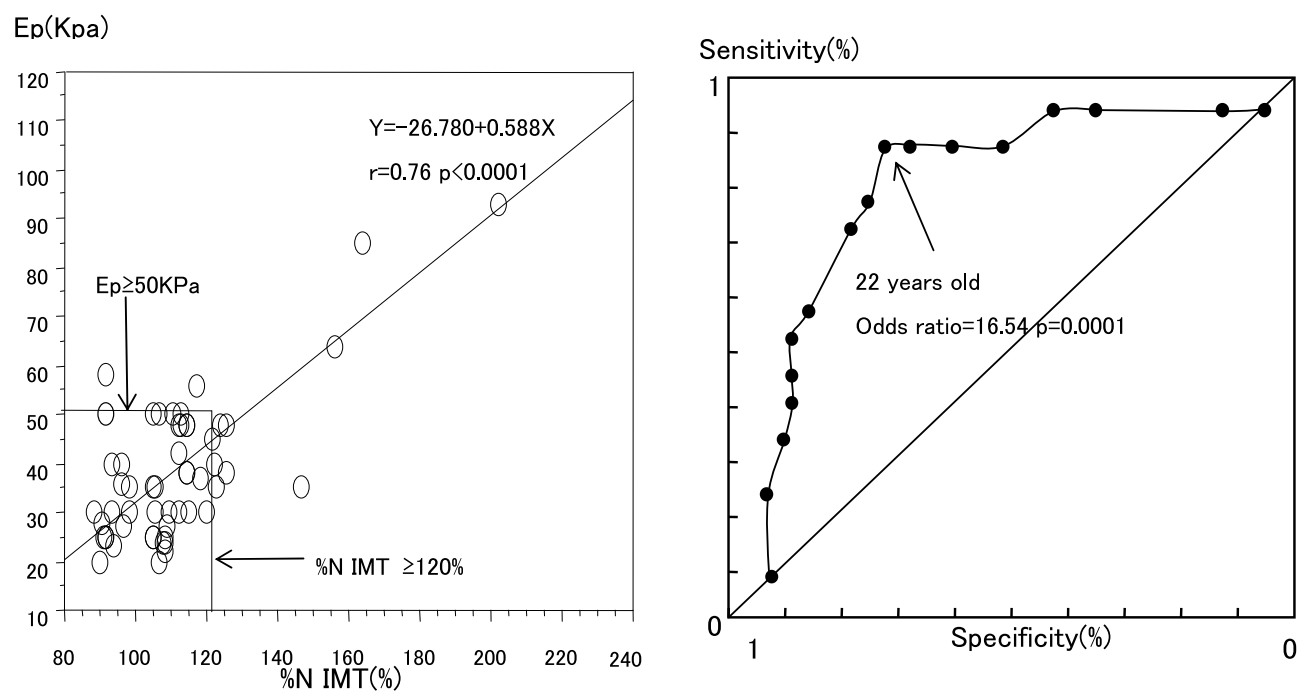

Fig. 7. Relationship between \% N IMT and Ep and ROC Curve in KD patients and Control Subjects. (Left) Regression line for all subjects is represented by a solid line. Arrows represent the cut-off points of $\% \mathrm{~N}$ IMT $\geq 120 \%$ and or Ep $\geq 50 \mathrm{KPa}$ as markers of subclinical atherosclerosis. (Right) Receiver-operating characteristic curve for the prediction of subclinical atherosclerosis against age in all subjects. Arrow indicates 22 years of age with a high odds ratio $(\mathrm{OR}=16.54, \mathrm{p}=0.0001)$.

\section{Future perspective: Detection of earlier atherosclerotic involvement in KD patients with CALs}

It remains unclear whether the vascular remodeling process of atherosclerosis in KD differs from that in adults. In particular, major concern has been raised as to whether the vessels with predisposed arteriosclerotic changes in KD patients with CALs may more easily develop atherosclerotic changes. Several studies showed that carotid IMT, a surrogate of atherosclerotic vessel wall change, is sensitive to risk intervention and constitutes a reliable indicator of clinical outcome in adult subjects, even in children with familial hypercholesterolemia (FH) (Jarvisalo et al., 2001). Furthermore, these changes in the arterial wall occur earlier in the carotid arteries than in the coronary circulation, making the carotid arteries an ideal site for detection of premature atherosclerotic disease (Bland et al., 1986). In addition, current study has demonstrated that textural changes in the carotid intima-media complex (IMC) by B-mode ultrasound were associated with early atherosclerotic involvement in patients with FH (Noto et al., 2011) (Figure 8). We speculated that changes in the textural characteristics of carotid IMC by B-mode ultrasound could be indicative of the earlier involvement of carotid atherosclerosis in KD patients with CALs. To test this hypothesis, we assessed the ultrasonic textural parameters of the IMC by first-order statistics, second-order statistics in KD patients with CALs without CRFs, age-matched healthy control subjects and age-matched FH subjects and findings were compared among the 3 groups. 


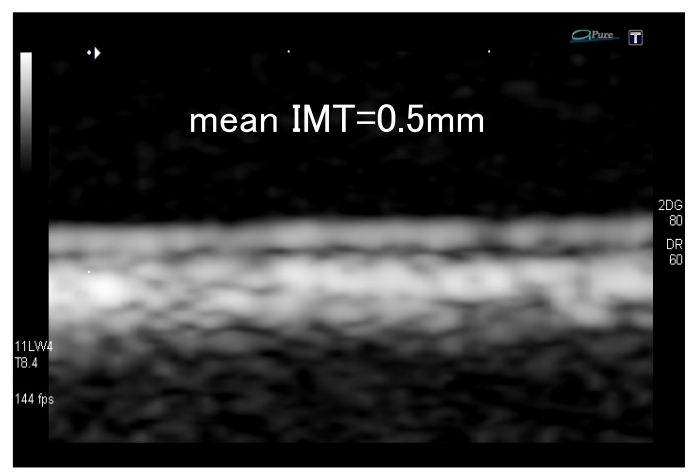

VS:1

normal wall

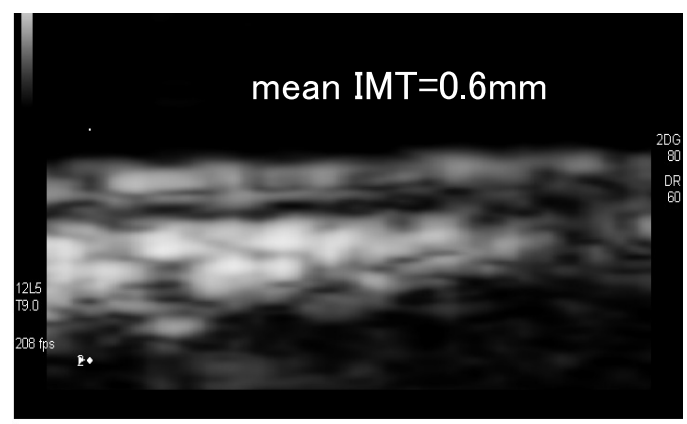

VS:2

proximal interface disrupted

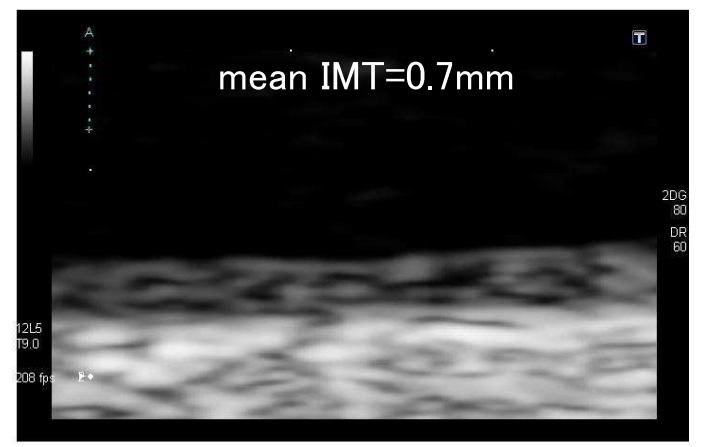

VS:3

intima-media granulation

Fig. 8. Representative typical Visual Scoring (VS) by magnified B-mode ultrasound images of the intima-media complex (IMC) in heterozygous familial hypercholesterolemia (FH). Visual Scoring 1: normal wall (intima media and adventitia clearly separated); Visual Scoring 2: proximal interface disrupted by hypoechoic components in the proximal echogenic layer; Visual Scoring 3: intima-media granulation with mixed hypoechoic and echogenic components. According to the increased IMT with medial infiltration of lipids, VS progresses from proximal interface disruption to intima-media granulation (Noto et al., J Am Soc Echocardiog 2011). 


\subsection{Study design}

To test the hypothesis that textural changes in the carotid intima-media complex (IMC) by Bmode ultrasound are associated with the earlier atherosclerotic involvement, 12 patients with KD and CALs without cardiovascular risk factors (mean age 20.5 years), 12 patients with heterozygous familial hypercholesterolemia (FH) (mean age 18.6 years) and age-matched 9 healthy control subjects (Cont) were assessed for intima-media thickness (IMT), first- and second-order statistics, and plot profile curve (PC) for regional analysis of the alteration of gray value in IMC, and were compared among 3 groups.

(a)

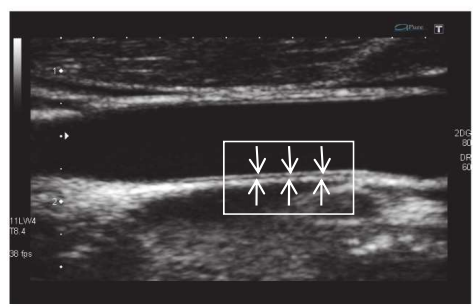

(b)

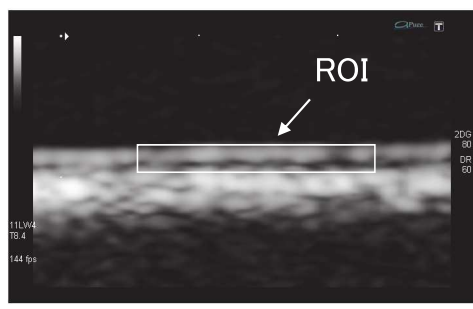

(c)

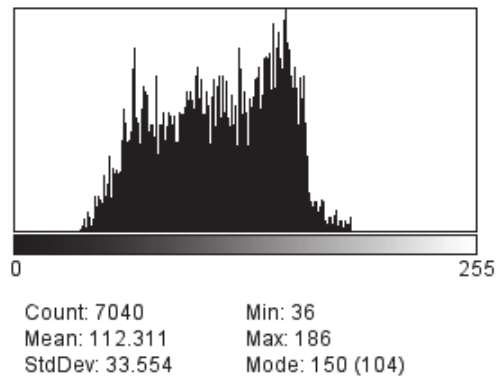

(d)

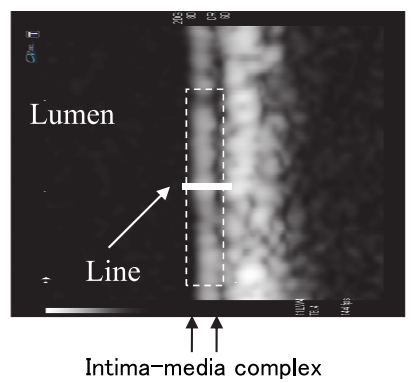

(e)

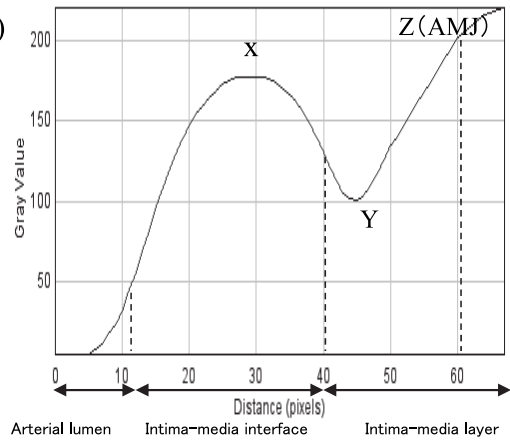

$X$ : maximum gray value of the intima-media

$Y$ : minimum gray value of the intima-media layer

$Z$ : gray value of the adventitia-media junction

AMJ: adventitia-medial junction

Fig. 9. Measurement of the textural parameters and gray value by plot profile curve (a) Conventional B-mode image of common carotid artery (CCA). Rectangle indicates the magnified portion around the far wall of CCA. (b) Maximal magnified B-mode image $(\times 8.6)$. Rectangle indicates the region of interest (ROI) in the far wall of the intima-media complex (IMC). (c) Histogram of ROI. This feature was automatically extracted by Image J $1.38 x$ software. $($ d) Maximal magnified B-mode image $(\times 8.6)$. Line indicates the portion of measurement of plot profile curve located center of ROI in the far wall of the intima-media complex (IMC). (e) Plot profile curve of gray values of the pixels along a $0.7 \mathrm{~mm}$ line over the IMC. 


\subsection{Results}

The mean IMT was significantly higher in FH $(p<0.0001)$ and KD $(p=0.005)$ than Cont. KD showed significantly higher gray-scale median (GSM) than FH $(p=0.027)$ and Cont $(p=0.048)$, however, there were no significant differences in Skewness, Kurtosis and Contrast among 3 groups. While, KD $(\mathrm{p}<0.0001)$ and $\mathrm{FH}(\mathrm{p}=0.004)$ showed significantly higher Entropy than Cont. Furthermore, KD $(p=0.0041)$ and FH $(p=0.001)$ showed significantly lower Angular second moment (ASM) than Cont, but no significant difference was found between KD and FH. As for PC, KD showed significantly higher gray value both at the minimum point in IMC $(p<0.01)$ and at the adventitia-media junction $(p<0.01)$ than $\mathrm{FH}$ and Cont. These findings demonstrate that higher GSM in KD may indicate alteration of tissue component and heterogeneity IMC, suggesting sclerotic vascular remodeling after vasculitis. In contrast, decreased GSM may indicate atherotic vascular remodeling often observed in $\mathrm{FH}$ patients.

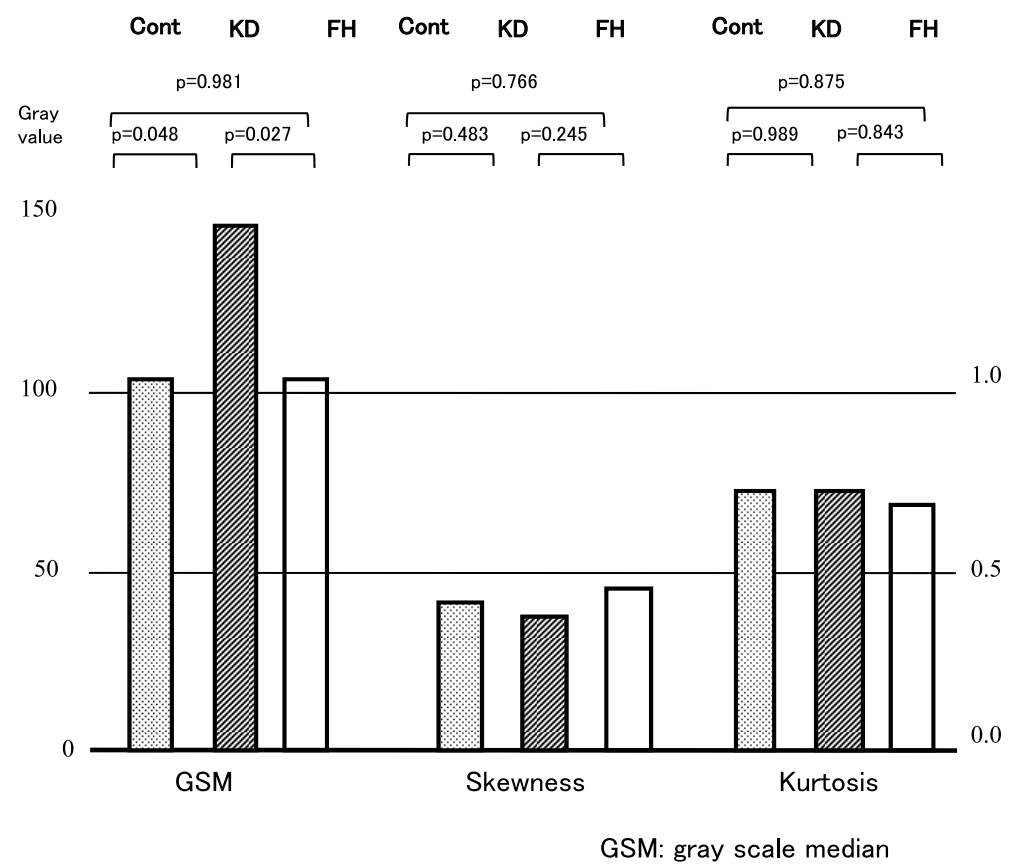

Fig. 10. Histogram variables among KD patients, Familial hypercholesterolemia, and control subjects.

\section{Conclusion}

We reviewed the recent several problems concerning with the propensity of atherosclerosis in patients long after KD. Among KD patients with CALs, impairments of coronary artery structure and function have been well documented. However, it is still too early to determine the conclusion whether post-KD patients in whom coronary abnormalities were never detected are at risk for premature atherosclerosis later in adulthood until the early 
Japanese cohort reaches middle and older age. To date, all patients with a history of KD should be carefully assessed for factors promoting atherosclerosis, including hypercholesterolemia, obesity, systemic hypertension, and smoking.

\section{References}

Burns JC, Shike H, Gordon JB, Malhotra A, Schoenwetter M, \& Kawasaki T. (1996). Sequelae of Kawasaki disease in adolescents and young adults. J Am Coll Cardiol, Vol. 28, pp. 253-257.

Cheung YF, Young TC, Ho MH, \& Chau AK. (2004). Novel and traditional cardiovascular risk factors in children after Kawasaki disease. J Am Coll Cardiol, Vol. 43, pp. 120124.

Cheung YF, Ho MH, Tam SCF, \& Young TC. (2004). Increased high sensitivity C reactive protein concentrations and increased arterial stiffness in children with a history of Kawasaki disease. Heart, Vol. 90, pp. 1281-1285.

Cheung YF, Wong SJ, \& Ho MH. (2007). Relationship between carotid intima-media thickness and arterial stiffness in children after Kawasaki disease. Arch Dis Child, Vol. 92, pp. 43-47.

Cheung YF, O K, Woo CW, Armstrong S, Siow YL, Chow P, \& Cheung EW. (2008). Oxidative stress in children late after Kawasaki disease: relationship with carotid atherosclerosis and stiffness. BMJ Pediatrcs, Vol.8, pp. 20.

Della Pozza R, Bechtold S, Urshel S, Kozlik-Feldmann R, \& Netz H. (2007). Subclinical atherosclerosis, but normal autonomic function after Kawasaki disease. J Pediatr, Vol. 151, pp. 239-43.

Dhillon R, Clarkson P, Donald AE, Powe AJ, Nash M, Novelli V, Dillon MJ, \& Deanfield JE. (1996). Endothelial dysfunction late after Kawasaki disease. Circulation, Vol. 94, pp. 2103-2106.

Fukazawa R, Ikeman E, Watanabe M, Hajikano M, Kamisago M, Katsube Y, Yamauchi H, Ochi M, \& Ogawa S. (2007). Coronary artery aneurysm induced by Kawasaki disease in children show features typical senescence. Circ J, Vol. 71, pp. 709-715.

Griending KK, \& FitzGerald GA. (2003). Oxidative stress and cardiovascular injury: Part 2: Animal and human studies. Circulation, Vol. 108, pp.2034-2040.

Hamaoka A, Hamaoka K, Yahata T, Fujii M, Ozawa S, Toiyama K, Nishida M, \& Itoi T. (2010). Effects of HMG-CoA reductase inhibitors on continuous post-inflammatory vascular remodeling late after Kawasaki disease J Cardiol, Vol. 56, pp. 245-253.

Iemura M, Ishii M, Sugimura T, Akagi T, \& Kato H. (2000). Long-term consequences of regressed coronary aneurysms after Kawasaki disease: vascular wall morphology and function. Heart, Vol. 83, pp. 307-311.

Ikemoto Y, Ogini H, Teraguchi M, \& Kobayashi Y. (2005). Evaluation of preclinical atherosclerosis by flow-mediated dilatation of the brachial artery and carotid artery analysis in patients with a history Kawasaki disease. Pediatr Cardiol, Vol. 26, pp. $782-786$.

Jarvisalo MJ, Jartti L, Nanto-Saloren K, Irjala K, Ronnemaa T, \& Hartiala JJ. (2001). Increased aortic intima-media thickness: a marker of preclinical atherosclerosis in high-risk children. Circulation, Vol. 104, pp.:2943-2947. 
Kato H, Sugimura T, Akagi T, Sano N, Hashino K, Kazunc T, Eto G, \& Yamakawa R. (1996). Long-term consequences of Kawasaki disease: A 10- to 21-year follow-up study of 594 patients. Circulation, Vol. 94, pp. 1379-1385.

Kawasaki T. (1967). Acute febrile muco-cutaneous lymph node syndrome in youngchildren with unique digital desquamation. Jpn J Allergol, Vol.16, pp. 178-222.

McCrindle BW, McIntyre S, Kim C, Lin T, \& Adeli K. (2007). Are patients after Kawasaki disease at increased risk for accelerated atherosclerosis? J Pediatr, Vol. 151, pp. 244248.

Mitani Y, Sawada H, Hayakawa H, Aoki K, Ohashi H, \& Matsumura M. (2005). Elevated levels of hight-sensitivity C-reactive protein and serum amyloid-A late after Kawasaki disease: association between inflammation and coronary sequelae in Kawasaki disease. Circulation, Vol. 111, pp. 38-43.

Mitani Y, Ohashi H, Sawada H, Ikeyama Y, Hayakawa H, Takabayashi S, Maruyama K, Shimpo H, \& Komada Y. (2009). In vivo plaque composition and morphology in coronary artery lesions in adolescents and young adults long after Kawasaki disease: a virtual histology intra vascular ultrasound study. Circulation, Vol. 119, pp. 2829-36.

Nakamura Y, Yashiro M, Uehara R, Sadakane A, Chihara I, Aoyama Y, Kotani K, \& Yanagawa H. (2010). Epidemiologic features of Kawasaki disease in Japan: results of the 2007-2008 nationwide survey. J Epidemiol, Vol.20, pp. 302-307.

Naoe S, Takahashi K, Masuda H, \& Tanaka N. (1991). Kawasaki disease. With particular emphasis on arterial lesions. Acta Pathol Jpn, Vol. 41, pp. 785-97.

Negoro N, Nariyama J, Nakagawa A, Katayama H, Okabe T, Hazui H, Yokota N, Kojima S, Hoshiga M, Morita H, Isihara T, \& Kanafusa T. (2003). Successful catheter interventional therapy for acute coronary syndrome secondary to Kawasaki disease in young adults. Circ J, Vol. 67, pp. 362-365.

Newberger JW, Burns JC, Beiser AS, \& Loscalzo J. (1991). Alterd lipid profile after Kawasaki syndrome. Circulation, Vol. 84, pp. 625-631.

Niboshi A, Hamaoka K, Sakata K, \& Yamaguchi N. (2008). Endothelial dysfunction in adult patients with a history of Kawasaki disease. Eur J Pediatr, Vol. 167, pp. 189-196.

Noto N, Okada T, Yamasuge M, Taniguchi K, Karasawa K, Ayusawa M, Sumitomo N, \& Harada K. (2001). Noninvasive assessment of the early progression of atherosclerosis in adolescents with Kawasaki disease and coronary artery lesions. Pediatrics, Vol. 107, pp. 1095-99.

Noto N, Okada T, Karasawa K, Ayusawa M, Sumitomo N, Harada K, \& Mugishima H. (2009). Age-related acceleration of endothelial dysfunction and subclinical atherosclerosis in subjects with coronary artery lesions after Kawasaki disease. Pediatr Cadiol, Vol. 30, pp. 262-268.

Noto N, Okada T, Abe Y, Miyashita M, Kanamaru H, Karasawa K, Ayusawa M, Sumitomo N, \& Mugishima H. (2011). Changes in the textural characteristics of intima-media complex in young patients with familial hypercholesterolemia: Implication for visual inspection on B-mode ultrasound. J Am Soc Echocardiogr, Vol. 24, pp. 438443.

Okada T, Karada K, \& Okuni M. (1982). Serum HDL-cholesterol and lipoprotein function in Kawasaki disease (acute mococutaneous lymph node syndrome). Jpn Circ J, Vol. 46, pp. $1039-1044$. 
Ooyanagi R, Fuse S, Tomita H, Takamuro M, Horita N, Mori M, \& Tsutsumi H. (2004). Pulse wave velocity and ankle brachial index in patients with Kawasaki disease. Peditr Int, Vol. 46, pp. 398-402.

Ozawa S, \& Hamaoka K. (2006). HMG-CoA refuctase inhibitors are effective for prevention of acute coronary injury in a rabbit model of Kawasaki disease. J Am Coll Cadiol, Vol. 46(Suppl A), pp.244A.

Sasaguri Y, \& Kato H. (1982). Regression of aneurysms in Kawasaki disease: a pathological study. J Pediatr, Vol. 100, pp. 225-231.

Suzuki A, Kamiya T, Arakaki Y, Kinoshita Y, \& Kimura K. (1994). Fate of coronary arterrial aneurysms in Kawasaki disease. Am J Cardiol, Vol. 74, pp. 822-824.

Suzuki A, Kamiya T, Tsuda E, \& Shinya T. (1997). Natural history of coronary arterial lesions in Kawasaki disease. Prog Pediatr Cardiol, Vol. 6, pp. 211-218.

Suzuki A, Miyagawa-Tomita S, Komatsu K, Nishikawa T, Sakomura Y, Horie T, \& Nakazawa M. (2000). Active remodeling of the coronary arterial lesions in the late phase of Kawasaki disease: Immunohistochemical study. Circulation, Vol. 101, pp. 2935-41.

Suzuki A, Miyagawa-Tomita S, Komatsu K, Nakazawa M, Fukaya T, Baba K, \& Yutani C. (2004). Immunohistochemical study of apparently intact coronary artery in a child after Kawasaki disease. Pediatrics International, Vol. 46, pp. 590-596.

Strong WB, Deckelbaum RJ, Gidding SS, Kavey RE, Washinton R, Wilmore JH \& Perry CL. (1992). Integreted cardiovascular health promotion in childhood. A statement for health profrssionals from the subcommittee on atherosclerosis and hypertension in childhood of the council on cardiovascular disease in the young, American Heart Association. Circulation., Vol. 85, pp. 1638-50.

Takahashi K, Oharaseki T, \& Naoe S. (2001). Pathological study of postcoronary arteritis in adolescents and young adults: with reference to the relationship between sequelae of Kawasaki disease and atherosclerosis. Pediatr Cardiol, Vol. 22, pp. 138-142.

Taubert KA, Rowley AH, \& Shulman ST. (1991). Nationwide survey of Kawasaki disease and acute rheumatic fever. J Pediatr, Vol. 119, pp. 279-282.

Tsuda E, Abe T, Tamaki W. (2011). Acute coronary syndrome in adult patients with coronary artery lesions caused by Kawasaki disease: review of case reports. Cardiol Young, Vol 21, pp.74-82.

Yamakawa R, Ishii M, Sugimura T, Akagi T, Eto G, Iemura M, Tsutsumi T, \& Kato H. (1998). Coronary endothelial dysfunction after Kawasaki disease: evaluation by intracoronary injection of acetylcholine. J Am Coll Cardiol, Vol.31, pp.1074-1080. 


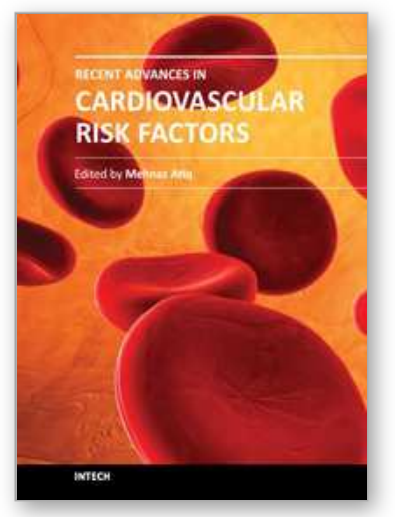

\author{
Recent Advances in Cardiovascular Risk Factors \\ Edited by Prof. Mehnaz Atiq
}

ISBN 978-953-51-0321-9

Hard cover, 522 pages

Publisher InTech

Published online 21, March, 2012

Published in print edition March, 2012

Among the non-communicable diseases, cardiovascular disorders are the leading cause of morbidity and mortality in both the developed and the developing countries. The spectrum of risk factors is wide and their understanding is imperative to prevent the first and recurrent episodes of myocardial infarction, stroke or peripheral vascular disease which may prove fatal or disabling. This book has tried to present an update on risk factors incorporating new research which has thrown more light on the existing knowledge. It has also tried to highlight regional diversity addressing such issues. It will hopefully be resourceful to the cardiologists, general practitioners, family physicians, researchers, graduate students committed to cardiovascular risk prevention.

\title{
How to reference
}

In order to correctly reference this scholarly work, feel free to copy and paste the following:

Nobutaka Noto and Tomoo Okada (2012). Premature Atherosclerosis Long After Kawasaki Disease, Recent Advances in Cardiovascular Risk Factors, Prof. Mehnaz Atiq (Ed.), ISBN: 978-953-51-0321-9, InTech, Available from: http://www.intechopen.com/books/recent-advances-in-cardiovascular-risk-factors/prematureatherosclerosis-long-after-kawasaki-disease

\section{INTECH}

open science | open minds

\section{InTech Europe}

University Campus STeP Ri

Slavka Krautzeka 83/A

51000 Rijeka, Croatia

Phone: +385 (51) 770447

Fax: +385 (51) 686166

www.intechopen.com

\section{InTech China}

Unit 405, Office Block, Hotel Equatorial Shanghai

No.65, Yan An Road (West), Shanghai, 200040, China

中国上海市延安西路65号上海国际贵都大饭店办公楼405单元

Phone: +86-21-62489820

Fax: +86-21-62489821 
(C) 2012 The Author(s). Licensee IntechOpen. This is an open access article distributed under the terms of the Creative Commons Attribution 3.0 License, which permits unrestricted use, distribution, and reproduction in any medium, provided the original work is properly cited. 